\title{
LITERATURDIDAKTIK IN FREMDKULTURELLER PERSPEKTIVE. AUSGEWÄHLTE ASPEKTE
}

\author{
Opus opificem probat \\ Meinem Meister und Freund, Herrn Prof. Dr. habil. Czesław Karolak, \\ mit Hochachtung und Dank
}

\section{Einführung}

Interkulturalität gehört zu den zentralen Fragen in den gegenwärtigen germanistischen Veröffentlichungen, und zwar in mehreren Perspektiven. Interkulturelle und kommunikative Aspekte findet man heutzutage sowohl in der Sprachdidaktik (besonders in der Fremdsprachendidaktik) als auch in der Literaturdidaktik, die fremdkulturelle Phänomene zu einem relevanten Gegenstand ihrer Forschungen gemacht hat. Der vorliegende Beitrag setzt sich in diesem Zusammenhang die drei folgenden Aufgaben zum Ziel:

- grundlegende Richtungen gegenwärtiger interkultureller Forschungen zu systematisieren, insbesondere in der fremdsprachendidaktischen Perspektive;

- ausgewählte Fragen der philosophischen Hermeneutik darzulegen;

- sowohl die wichtigsten Ziele als auch den potenziellen Forschungsgegenstand einer neuen literaturdidaktischen Teildisziplin, einer interkulturellen Texthermeneutik, zu bestimmen.

\section{Interkultureller und kommunikativer Ansatz als Forschungsproblem in der fremdsprachlichen Literaturdidaktik}

Interkulturalität als fremdsprachendidaktisches Phänomen ist eine der zentralen Fragen, auf die heutzutage fast alle Sprachdidaktiker in ihren Forschungen verweisen. Seitdem der kommunikative Ansatz den Fremdsprachenunterricht weltweit 
dominiert hat, gilt die sog. interkulturelle Kompetenz als eines der wichtigsten Unterrichtsziele, natürlich neben anderen Fertigkeiten und Fähigkeiten im Fremdsprachenerwerb. In der Forschung verbindet man den Begriff der interkulturellen Kompetenz auch mit der Fähigkeit oder sogar Fertigkeit von Kommunikationspartnern einer fremdkulturellen Begegnung, Differenzen zwischen der eigenen und der Zielkultur zu erkennen, potenziellen Missverständnissen vorzubeugen und damit die Interaktion problemlos zu gestalten. Zugleich werden auch in diesem Zusammenhang komplexe Lernziele des modernen Fremdsprachenunterrichts vorausgesetzt (Volkmann, 2002, S. 13):

- Kenntnisse der eigenen Kultur und ihrer kulturellen Codes;

- Wissen um sog. „fremde Heterostereotype“ (subjektive Einschätzung von Mitgliedern einer anderen Kultur);

- Wissen um konventionelle Stereotype, die die Wahrnehmung der anderen Kultur steuern;

- Kenntnisse der eigenen und fremden Kultur (auch Sub- und Alternativkulturen);

- keine unreflektierte Übernahme von Elementen der anderen Kultur;

- die Fähigkeit, kulturelle Barrieren bewusst zu überschreiten.

Eine erweiterte Auffassung der Rolle der interkulturellen Kompetenz im Fremdsprachenunterricht findet man auch bei Barkowski und Krumm (2010, S. 140), wo ihre emotionellen, kognitiven sowie sozialen Aspekte betont werden.

Leider wird dieser Terminus auch oft sehr weit aufgefasst und als eine Art „Wundermittel“ verstanden, das gegen alle Schwierigkeiten, die im Unterrichtsprozess anzutreffen sind, erfolgreich verwendet werden könnte. Bezeichnungen wie „,interkulturell“, „multikulturell“ oder die neuere Prägung „transkulturell““ beherrschen inzwischen viele Komplexe der modernen Fremdsprachendidaktik. So hören wir oft nicht nur von der ,interkulturellen Kompetenz und Kommunikation“, sondern auch z. B. vom ,,interkulturellen Lernen“, der ,interkulturellen Germanistik“, der ,multikulturellen Erziehung und Didaktik“ (Szeluga, 2004, S. 945), um nur einige besonders geläufige Bezeichnungen zu nennen. Das Hauptproblem des modernen Fremdsprachenunterrichts lautet allerdings: Wie sind diese interkulturellen Komponenten in wirkliche, reale Sprachfertigkeiten umzusetzen? Will man dieser Fragestellung näherkommen, so muss man auch die dem Begriff „Interkulturalität“ zugrunde liegenden Forschungen berücksichtigen.

Die interkulturelle Betrachtungsweise in der germanistischen Literaturdidaktik lässt sich auf das Konzept der „interkulturellen Germanistik“ und des Faches Deutsch als Fremdsprache zurückführen (siehe dazu u.a. Karolak, 1999, S. 24-26). In diesem Modell sollten in die praktische Schullehre landeskundliche und literaturwissenschaftliche Komponenten integriert werden, so dass angewandte Aspekte (z. B. Interpretation und Vermittlung fremdsprachlicher Literatur oder Verstehensstrategien literarischer Werke in fremdkultureller Perspektive) beider Disziplinen betont wurden. Das Fach Deutsch als Fremdsprache hat sich zum Hauptziel gesetzt, 
zwei Teilkomponenten zu verbinden: die philologische und die fremdkulturelle, die wiederum landes- und kulturkundliche, literaturdidaktische und kulturvergleichende Inhalte umfassen sollte. Die kulturräumliche Dimension hat mit der Zeit an Bedeutung gewonnen und sich zur Kulturkomparatistik etabliert. In diesem Kontext kann man Beiträge u. a. von Ehlich, Henrici, Krumm, Neuner oder Weinrich nennen. Für Karolak (1999, S. 8-10) ist die fremdkulturelle Distanz eines fremdsprachigen Rezipienten keine potenzielle Gefahr der Ausgrenzung dieser Lesergruppe, sondern eher eine große Chance, die kulturräumliche Fremde als Bereicherung der Textrezeption zu verwerten.

Moderne interkulturelle Forschungen lassen sich grundsätzlich in zwei wesentliche Bereiche einteilen: es sind einerseits sog. ,intercultural studies“ und „multicultures education“, die hauptsächlich auf interkulturellen Dialog und Fremdverstehen ausgerichtet sind und den Forschungsgegenstand einer gegenwartsorientierten Kulturwissenschaft im weiteren Sinne (aber auch z. B. der Ethnolinguistik) bestimmen. Die andere Forschungsrichtung konzentriert sich auf die sog. „Didaktik der Fremde“ oder „Didaktik der Fremdheitskompetenz“, die als ein multidimensionales und interdisziplinär angelegtes Fach über die traditionelle Fremdsprachendidaktik deutlich hinausgeht (Szeluga, 2004a, S. 948), und deren Kern der sog. „,kulturelle Code“ bestimmt. Dieser Terminus orientiert sich an den modernen Kognitionswissenschaften (Linguistik und Psychologie) und bedeutet sowohl die Gesamtheit aller Kategorisierungs- und Schematamuster in all ihren Formen als auch alle kognitiv vorgeprägten Strukturen, die das menschliche Verarbeitungspotenzial mit seinen Verstehensleistungen organisieren und auf diese Art und Weise unsere Wirklichkeitswahrnehmung determinieren.

Der kulturelle Code wird in den kognitiven Arbeiten mit der Kategorisierung und mit der Bildung von mentalen Repräsentationen im menschlichen Gehirn in Verbindung gesetzt. Das Verstehen beansprucht die Kenntnis sowohl der allgemeinen Ebene der Bildung von mentalen Repräsentationen, die für alle Sprecher einer Sprache mehr oder weniger ähnlich ist, als auch der individuellen Ebene, die als kognitive Leistung eines einzelnen Sprechers aufzufassen und daher für andere Sprecher als solche unerreichbar ist. Beide Ebenen durchdringen sich mehrdimensional, weil gemeinsame soziokulturelle Kontexte des sprachlichen Handelns und der gemeinsame Sozialisationsrahmen nur im konkreten sozialen Umfeld und in sozialer Praxis realisiert werden können (Szeluga, 2003b, S. 840). Ein sprachlicher Ausdruck gewinnt seine Bedeutung, oder genauer ausgedrückt sein Bedeutungsspektrum aus der theoretisch unbegrenzten Zahl von jeweiligen Zusammenhängen und mitgemeinten Kontexten aller Sprecher einer Sprache.

Dieser Prozess kompliziert sich weitgehend im Falle eines fremdsprachigen Sprechers, für den dieser gemeinsame Sozialisationsrahmen schwer oder kaum zugänglich ist.

In Anlehnung z. B. an Habermas (1986, S. 59) wissen wir jedoch, dass eine Fremdsprache nur in dem Ausmaß zu erwerben ist, in dem wir den Sozialisations- 
prozess, den die Muttersprachler erfahren haben, einholen können (auch wenn es nur virtuell geschehen kann). Natürlich müsste man in diesem Zusammenhang zwischen zwei Situationen unterscheiden: eine fremde Sprache kann entweder im Zielsprachenmilieu oder unter fremdkulturellen Bedingungen erlernt werden, doch in keinem dieser zwei Fälle ist es möglich, den muttersprachlichen Sozialisationsrahmen völlig einzuholen, was einen fremdsprachigen Sprecher von vornherein in eine andere Position versetzt als einen Muttersprachler. Daraus folgt eine erste Schlussfolgerung: Ein fremdsprachiger Leser rezipiert einen Text immer anders als ein muttersprachlicher, was allerdings keine negative Erscheinung zu sein braucht. Sich eine Sprache anzueignen heißt doch, nicht nur strukturelle Regeln des jeweiligen Sprachsystems zu erlernen (Grammatik und Lexik). Selbst die Beherrschung eines noch so umfangreichen außersprachlichen Wissens und noch so weitreichender Kenntnisse über den zu erlernenden Sprachraum (u. a. landeskundliche und kulturkundliche Informationen) ist nicht mit einer interkulturellen Kompetenz gleichzusetzen. Dieses Wissen ist zweifellos ihr immanenter Teil, doch über interkulturelle Kompetenz zu verfügen, bedeutet, diese komplexen Wissensbestände entsprechend anwenden zu können.

In diesem Zusammenhang ist in der Fremdsprachendidaktik der Begriff der „Fremdheitskompetenz" geprägt worden, die sowohl die interkulturelle als auch die kommunikative Kompetenz integrieren soll und methodologisch gesehen einen besonders umfangreichen und multidimensionalen Terminus bezeichnet. Dessen Ausgangspunkt bildet der Begriff der kommunikativen Kompetenz, der im Zentrum der wichtigsten fremdsprachendidaktischen Tendenzen der letzten Jahrzehnte steht. Unter mehreren Definitionen könnte man hier die Konzepte von Hymes (1972), Habermas (1981) und Wunderlich (1972) nennen (dazu mehr bei Szeluga, 2003a, S. 411-417), weil sie in den Forschungen die stärkste Rezeption gefunden haben. Kommunikative Kompetenz ist nach ihnen (wobei sie einzelne Akzente unterschiedlich gesetzt haben) als die Fähigkeit eines Sprechers aufzufassen, sich innerhalb einer Sprachgemeinschaft sozialkommunikativ angemessen zu verhalten, um bedeutungsvolle Äußerungen zu bilden und zu verstehen.

Der Begriff der „Fremdheitskompetenz“ umfasst bekanntlich mehrere Teilkompetenzen und Fähigkeiten, die den Begriff der kommunikativen Kompetenz weitgehend ergänzen. Als die drei wichtigsten erscheinen in diesem Kontext: Fachkompetenz, soziokulturelle Kompetenz und Handlungskompetenz. Fachkompetenz bedeutet sowohl Anwendung von Wissen und Kenntnissen als auch ein Verarbeitungssystem, dank dem dieses Wissen effizienter und rationeller zu organisieren ist. Im Fremdsprachenunterricht bezeichnet sie die Kenntnis der Grammatik, grammatischer Strukturen sowie außersprachliches Wissen über eine fremde Kultur. Handlungskompetenz (auch im Sinne einer Informations- und Medienkompetenz) ergänzt die Fachkompetenz um Fähigkeiten, die neue Informationen suchen und finden, analysieren und anwenden helfen, und begünstigt zugleich bilinguales Lernen, das 
wiederum mutter- und fremdsprachliche Strategien verbindet. Soziokulturelle Kompetenz wird auf affektiver Ebene wirksam und umfasst ethische Kompetenz, Umwelt-Kompetenz, ohne die ein kooperatives und soziales Handeln innerhalb einer Sprachgemeinschaft unmöglich wird (Szeluga, 2004a, S. 949).

Das Zeitalter der Globalisierung, der kulturelle Grenzen überschreitenden Kontakte und Erfahrungen, erfordert ohne Zweifel neue didaktische Konzeptionen sowie eine Präzisierung des bereits sehr geläufigen Begriffes ,interkulturelle Kompetenz“, und zwar nicht nur auf der deklarativen Ebene einer erwünschten Sprachkompetenz, sondern vor allem als einer kognitiven, stark internalisierten Leistung der Lerner.

\section{Interkulturalität in den hermeneutischen Forschungen}

Der Terminus des „kulturellen Codes“ wird in den kognitiven Untersuchungen mit dem Begriff der „kulturellen Metapher“ verglichen, die jeweilige Symbole, Ikonen und Metonymien in einer Sprache zum Ausdruck bringt und damit auch die Bedeutung einer Äußerung konstituiert (Szeluga, 2003b, S. 841). Ein fremdsprachiger Leser bedient sich zweier Systeme von Metaphern, wobei sich das muttersprachliche System und das fremdsprachliche überschneiden. Das Vorwissen des Rezipienten im Sinne von Gadamer, das in seiner Muttersprache verankert ist, prägt die Verstehensprozesse in der jeweiligen Fremdsprache, wobei diese Prozesse u. a. oft von Stereotypen und Vorurteilen über die fremde Kultur beeinflusst und mitgestaltet werden.

Textuelle Aussagen beschränken sich nicht nur auf direkte Kommunikation im Text, sondern implizieren zahlreiche „Tiefenstrukturen“, die meistens als soziokulturelle Kontexte verschlüsselt sind. Das Textverstehen und Textinterpretieren hängen davon ab, ob beide Partner (Autor und Leser) über gemeinsame Aussagenkontexte verfügen oder nicht. In diesem kommunikativen Wechselspiel werden bekannte Interpretationsschemata umgesetzt, und neue Inhalte schaffen auch neue Auslegungsmöglichkeiten. Das interkulturelle Gespräch stellt kulturrelevante Elemente zur Diskussion, die mit muttersprachlichen Inhalten verglichen und neu definiert werden. Eine neue ästhetische Erfahrung, die als Ergebnis dieses Prozesses anzusehen ist, bietet dem fremdsprachigen Leser neue Wege zum Verstehen und die Grenzen zwischen dem Eigenen und dem Fremden verschieben sich dabei, weil er muttersprachliche Konnotationen relativieren muss. Es bedeutet gleichzeitig auch eine Chance, über diese hinauszugehen und das Fremde aus einer relativierten, d. h. bikulturellen Perspektive interpretieren zu können (Szeluga 2003b, S. 843-844).

Das Konzept einer „interkulturellen Texthermeneutik“ gründet sich einerseits eben auf den oben skizzierten interkulturellen Forschungen und andererseits auf der hermeneutischen Tradition in der Literaturwissenschaft, besonders der philosophischen Hermeneutik von Gadamer (2003, S. 114). Seine Textauffassung bedeutet 
nicht, dass er den Text aus rein sprachlicher, linguistischer Sicht betrachtet, weil er die formorientierte Auslegung ablehnt. Aus hermeneutischer Perspektive ist ein Text nur ein Zwischenprodukt, eine Phase im Verstehensprozess. Der Zusammenhang zwischen dem Text und seiner Interpretation hat einen konstitutiven Charakter und wird immer von der konkreten Situation motiviert. Die Interpretation und das Verstehen werden gleichzeitig im Leseakt „,eingeschaltet“, sie kommen miteinander ins Gespräch. Mit dieser Einstellung wendet sich Gadamer von der „,romantischen Hermeneutik" ab. Die Fremdsprachlichkeit bezeichnet er als

\footnotetext{
einen gesteigerten Fall von hermeneutischer Schwierigkeit, d. h. von Fremdheit und Überwindung derselben. (....) Das Bespiel des Übersetzers, der die Kluft der Sprachen zu überwinden hat, lässt die Wechselbeziehung besonders deutlich werden, die zwischen dem Interpreten und dem Text spielt und die der Wechselseitigkeit der Verständigung im Gespräch entspricht" (Gadamer, 1975, S. 365).
}

Fremdsprachlichkeit und Verstehen weisen (mit einigen Einschränkungen) offensichtlich viele Gemeinsamkeiten auf. Die Fremdheit, die einen Text geradezu unverständlich macht, muss von dem Interpreten aufgehoben werden, sonst spricht er dazwischen, weil der Text seine Funktion, verstanden zu werden, nicht erfüllt. Die Interpretation schafft demnach keinen Text, sondern sie dient ihm, indem sie das überwindet, was im Text fremd ist, und damit hilft sie auch dem Leser beim Verstehen. Die Perspektive des Textes und die des Lesers verschmelzen, was Gadamer (1960, S. 289) „Horizontverschmelzung“ nennt. Eine besondere Textart sind in diesem Zusammenhang literarische Texte. Ein literarischer Text verweist nicht auf den primären Sprechakt, sondern er schafft selbst neue Sprechakte und verfügt damit über eine eigene Authentizität. Mitbedeutungen eines literarischen Textes verschaffen der Sprache ihren literarischen Reichtum, durch welchen sie einen höheren Bedeutungskontext bekommt (Gadamer, 2003, S. 127-132).

Den offenen Charakter der literarischen Texte bezeichnen viele Autoren auch anders: es sind „Leerstellen“ bei Iser, „Unbestimmtheitsstellen“ bei Ingarden, „Spielraummerkmale“ bei Groeben, ,semantische Offenheit" bei Steinmetz oder „Zwischenräume“ bei Krusche (dazu Szeluga, 2004b, S. 654-655). Der Autor und der Leser setzen eine Reihe von Kompetenzen voraus, die jedoch einander nicht unbedingt gleichen müssen, was zu potenziellen Interpretationsschwierigkeiten führen kann. Ein fiktionaler Text steht zwischen Vergangenheit und Zukunft und inszeniert seinen Code in Form von Signifikanten, die dann vom Leser als Rückmeldungen empfangen werden. Die Bedeutung kommt dann zu Stande, wenn aus einer Fülle von Bedeutungsmöglichkeiten eine konkrete aktualisiert wird. Die Kommunikation Text-Leser ist daher keine symmetrische, und in den modernen Werken spricht man sogar von der „Spaltung im Leser selbst“ (Iser, 1976, S. 251), die als Konfrontation seines bisherigen Bewusstseins von Ich und Welt mit der aktuellen Textinterpretation auszulegen sei. 
Die Unterrichtspraxis zeigt, dass die Verwendung von literarischen Texten beim Fremdsprachenlernen sowohl den Lernern als auch den Lehrenden so enorm große Schwierigkeiten bereitet, dass man nicht selten auf den Einsatz von Literatur überhaupt verzichtet. Die Schüler, die zum ersten Mal mit fremdsprachiger Literatur konfrontiert werden, erleben einen tiefen Schock. Das kann damit erklärt werden, dass im Unterricht eine Fremdsprache als System vermittelt wird und eine ,geordnete" Welt der Erfahrungen liefert. Im fiktiven Text begegnet der Schüler dagegen einer komplexen Welt der Empfindungen, und diese beiden Erfahrungen werden im Fremdsprachenunterricht einander gegenübergestellt. Eine gut überlegte Wahl der zu behandelnden Texte könnte jedoch diesem Schock oft erfolgreich vorbeugen.

Die Art und Weise der Wahrnehmung von literarischen Texten korrespondiert jedoch auch mit den kommunikativen Zielen der Sprachdidaktik. Literatur, die als Mimesis der gesellschaftlichen Praxis, Zufluchtsort für eine entfremdete Gesellschaft oder Probierfeld der Zukunft verstanden wird, leistet einen beträchtlichen Beitrag zur Entfaltung des Leseverstehens. Fremdkulturelle Literaturerfahrung versteht sich in diesem Zusammenhang als ein dynamischer und viel schwierigerer Prozess der Textdekodierung, der sich mindestens in drei Dimensionen vollzieht:

1. der Sprachcode als solcher muss dekodiert und verstanden werden;

2. der literarische Code beansprucht vom Leser eine Kompetenz, den ästhetischen Code der Literatur entsprechend zu interpretieren;

3. der fremdsprachige Code und der fremdkulturelle Rahmen müssen mit den muttersprachlichen soziokulturellen Kontexten verglichen und entsprechend „ausgewertet“" werden.

Diese Trichotomie der fremdkulturell bedingten Literaturwahrnehmung baut das in der Rezeptionsästhetik bekannte klassische Interpretationsmodell eines literarischen Werkes wesentlich aus. Viele Dimensionen dieses Prozesses werden dabei dem Leser nähergebracht.

Eine zweite Schlussfolgerung in diesen Überlegungen kann folgendermaßen formuliert werden: Ein fremdsprachiger Leser wird bei der Lektüre einer unvergleichbar komplizierteren Aufgabe als ein muttersprachlicher Rezipient ausgesetzt und kann sich dabei eines viel kleineren Interpretationsinstrumentariums bedienen.

\section{4. „Distanz" und „Fremde“ als zentrale Kategorien einer kulturell bedingten Literaturwahrnehmung}

Wenn man fremdsprachige Literatur liest, verändert man wesentlich seine Leseerwartungen. Man ist viel häufiger bereit, allerhand Unverständlichkeiten zu begegnen, es wird weniger Bestätigung erwartet, als das bei der Lektüre von muttersprachlichen Texten der Fall ist. Eine viel wichtigere Rolle spielen solche Größen wie Überraschung, Neugier oder auch Angst. 
Der Leser ist viel weniger vom Nichtverstehen betroffen; er ist auch geneigt, viele „Leseumwege“ zu gehen, um den Sinn des Textes zu konstituieren und zum Verstehen zu kommen. Vorurteile oder gar stereotype Vorstellungen gegenüber der uns fremden Kultur schaffen eine Atmosphäre der Gelassenheit. Erleben wir eine Inkompetenz, ein Versagen, weil unsere Kompetenz in der Zielsprache und Zielkultur unzulänglich ist, so werden sie nicht nur leichter genommen, sondern oft als etwas „Normales“ akzeptiert. Es ist ja schließlich eine „fremde Literatur“, d. h. sie ist als solche wegen kultureller Ferne immer schwieriger zu verstehen.

Der Lesevorgang verläuft dabei viel individueller und damit auch intensiver. Eine individuelle Leseerfahrung wird, vom Begriff der Distanz aus betrachtet, zu einem exemplarischen ästhetischen Erlebnis. Im Lese-Akt setzen wir bekanntlich die Außenwelt in Innenwelt um, wir erfahren uns selbst als Subjekte. Der Leseprozess ist eine Verflechtung von Fremd- und Selbsterfahrung des Lesers, die ihren Ausdruck im Dialog zwischen dem Lesenden und dem Text findet. Im Gespräch über Leseerfahrung kommen wir jedoch leicht in einen Zwiespalt. Unsere eigene Texterfahrung, die wir als einen Bestandteil unserer Identität ansehen, steht im Widerspruch zu potenziellen Aussagen anderer über den Text. Sie sind als solche wiederholbar, und dieses Merkmal lässt uns vor der Kommunikation über das Gelesene, vor dem gegenseitigen Lesedialog zurückschrecken. Wenn aber das Gespräch die von uns gemachten Erfahrungen nicht in Frage stellt, sondern sie ergänzt und fortsetzt, dann bekräftigt sich auf Seite des Lesers die Tendenz, diese „Flucht nach innen“ zu überwinden. Krusche (1981, S. 4) nennt diese Erscheinung „,interne Verschiebung". Ein Vergleich von verschiedenen Leseerfahrungen, die im interkulturellen Austausch gemacht wurden, leistet die Konkretisierung des Textsinnes. Unser Selbstbewusstsein als Leser kommt auch in allen Inhalten zur Sprache, die soziohistorisch bedingt sind. Wir werden uns in diesem Prozess unserer „Zugehörigkeit“ bewusst, und zwar in allen ihren Perspektiven: der sozialen, kulturellen und schließlich nationalen. Im interkulturellen literarischen Gespräch lässt sich oft die Tendenz der Teilnehmer beobachten, auf den jeweiligen gesellschaftlichen und kulturhistorischen Kontext keinen Bezug zu nehmen (besonders in einer direkten Form). Stehen wir als Gesprächsteilnehmer den Vertretern anderer Kulturen gegenüber, so sind wir selten bereit, unsere sozio-kulturelle Prägung zum Ausdruck zu bringen. Diese Tendenz wiederum, unsere eigene Leserposition unabhängig von soziokulturellen und gesellschaftlichen Kontexten öffentlich zu äußern, nennt Krusche (1981, S. 6) dagegen „externe Verschiebung“. Zwischen diesen beiden Polen verläuft das interkulturelle Gespräch über Leseerfahrungen.

In der Vermittlung von Literatur über kulturelle Grenzen hinweg wird als eines der primären Lernziele die Formulierung von unterschiedlichen Textinterpretationen beansprucht. Im Gegensatz zu muttersprachlichen literarischen Gesprächen geht es im interkulturellen Lesedialog nicht darum, die zwischen den Gesprächsteilnehmern liegende Ferne (als kulturell-historische Vorprägung der Rezeptionsfähigkeit begrif- 
fen) zu überbrücken oder aufzuheben. Fremdsprachige Literatur zu vermitteln und zu verstehen, heißt vielmehr, abweichende Äußerungen und Einstellungen zur Sprache zu bringen und sie zu konkretisieren. Die Dichotomie zwischen den muttersprachlichen und nicht-muttersprachlichen Lesenden lässt die zahlreichen Differenzen in ihren Lesepositionen formulieren (Krusche, 1981, S. 14):

- im kulturhistorischen, besonders literaturhistorischen Vorwissen,

- als Folge der Prägung durch verschiedene Sprachstrukturen,

- als Folge der Prägung durch verschiedene gesellschaftsgeschichtliche und sozio-politische Entwicklungen,

- als Folge der Prägung durch verschiedene Kulturtraditionen (Religion, Kunst, Philosophie),

- als Folge der Prägung durch unterschiedliche primäre Erfahrungen aus Klima, Landschaft, Natur mit ihren Nachwirkungen für die Vorstellungskraft der Leser,

- im Verhältnis zur Tradition, insbesondere der literarischen Tradition, zum Begriff der eigenkulturellen Identität/Bewusstsein (Zugehörigkeit/Nichtzugehörigkeit zu einem großen Kulturraum, wie z. B. Europa, Asien, Amerika).

Eine Fremdkultur wird immer vor dem Hintergrund der eigenen Kultur kennengelernt (ähnlich wie eine Fremdsprache im Spiegel der eigenen Sprache gelernt wird). Wenn sie jedoch mit eigenem Kulturbesitz rationell verglichen werden soll, muss sie auch von der eigenen Position aus in Besitz genommen werden. Es geschieht schrittweise, mit beträchtlichen Spannungen in den Lernern, die aus der Gefährdung der eigenen Kulturerfahrung resultieren. Hoffnungen und Ängste, die diesen Prozess begleiten, sind eine starke kompensatorische „Anziehungskraft" und lösen Motivation beim Lernen und Kennenlernen des Fremden aus. Das gegenseitige Vergleichen von beiden Polen (das Eigene - das Fremde) ist von der Position des Eigenen bestimmt, und von ihr wird auch ausgegangen. Das Fremde ist dagegen die Position, auf die man sich hinbewegt. Die Gefährdung des Eigenen erfüllt dabei die Rolle einer Öffnung für neue Erfahrungen und als ihr Resultat eine Relativierung des eigenen Selbstbewusstseins.

Der fremdkulturelle Adressat bewegt sich nicht selten in einem anderen Sozialisationszusammenhang als sein muttersprachlicher Partner. Verlagern wir die Normen und Regeln unseres Denkens und kommunikativen Handelns aus unserer Kultur und Gesellschaft in eine fremde Kulturlage, so besteht auch die Gefahr, dass unser Verhalten negativ beeinflusst wird. Die kulturelle Ferne erreicht den Leser am schärfsten dort, wo die Verbindung zwischen sinnlichen Momenten eines literarischen Textes und denen des Lesers in Frage gestellt wird. Alle bedeutungsvollen Zusammenhänge, die über das faktische, inhaltliche Wissen hinausgehen und eine individuelle Erfahrung erzwingen, beanspruchen ein Können, das sich nur durch kulturelle, ästhetische Erfahrungen erwerben lässt. Nur ein entsprechend gewählter, relevanter literarischer Text vermittelt dieses Können in allen seinen Aspekten. Wenn er aus fremdkultureller Perspektive gelesen wird, so gerät er in eine räumliche 
und oft auch zeitliche Entfremdung und läuft dabei Gefahr, dass alle vielfältigen Konnotationen, die im eigenen Kulturraum ihre Vernetzung haben, weggelassen werden. Dieser Gefahr müssen sich Lehrkräfte als fremdkulturelle Mittler und Vermittler bewusst sein, auch wenn es viele didaktische Probleme im fremdsprachigen Literaturunterricht bereitet. Es lohnt sich sehr, und zwar der imaginativen Kraft literarischer Werke wegen.

\section{Interkulturelle Texthermeneutik. Einige Grundlagen und Forschungsziele}

Die Grundlagen der Fundierung einer interkulturellen Texthermeneutik weisen demnach einen multidisziplinären Charakter auf und lassen sich auf mehrere Wissensbereiche beziehen:

- auf die Kulturwissenschaften sensu stricto und ihre gegenwärtigen inter- oder transkulturellen Forschungen, im Sinne einer Art Kulturkomparatistik;

- auf die fremdsprachendidaktischen Tendenzen, die als „Didaktik der Fremde“ oder „Fremdheitskompetenz“ aufgefasst werden und einen erweiterten Begriff der interkulturellen Kompetenz beanspruchen;

- auf viele Aspekte der modernen, kognitiv ausgerichteten linguistischen Textanalyse, die Sprachverarbeitungsprozesse zu erläutern versuchen;

- auf die philosophische Hermeneutik in den literaturwissenschaftlichen Forschungen (besonders von Gadamer) und ihre Implikationen in der Wirkungsund Rezeptionsästhetik.

Solch eine umfangreiche theoretische Fundierung einer Subdisziplin der Literaturdidaktik resultiert aus zahlreichen Zielen, die sie zu realisieren vermag. Einen der ersten Versuche, sie zu formulieren, hat zu Beginn der 80er Jahre Schmidt unternommen (vgl. Karolak 2004, S. 237). Er differenziert detailliert innerhalb spezieller Lernziele:

- Förderung der sprachlichen Kompetenz (im rezeptiven und produktiven Bereich);

- Förderung der Fähigkeit, sich in konkreten sozialen Situationen erfolgreich zu verhalten (Schmidt nennt sie kommunikative Kompetenz, was heutzutage terminologisch gesehen etwas verwirrend klingen mag);

- Förderung der Fähigkeit zu metasprachlicher Paraphrase, Kondensation, Analyse und Kommentierung von Texten;

- Förderung der Bereitschaft und Fähigkeit, sich das Kontextwissen außerhalb des Unterrichts anzueignen.

Er unterscheidet weiterhin zwischen monologischen, dialogischen und polylogischen Kommunikationsformen und verschiedenen Kommunikationsarten mit ihren Funktionen. Der gegenwärtige Forschungsstand ermöglicht es uns, diese Ziele zu 
ordnen und sie in neue Kontexte zu stellen. Aus den oben angeführten Dimensionen der dreifachen Dekodierung (sprachlicher, literarischer und fremdsprachiger) folgen diese Komplexziele:

- Entwicklung und Ausbau einer umfassenden kommunikativen Kompetenz, verstanden als die Fähigkeit des Lerners, den jeweiligen Text rezeptiv, reproduktiv und schließlich auch produktiv als Lernobjekt zu betrachten;

- Kenntnis und Anwendung von ästhetischen Sprachmitteln (literarischer Code), die für die Interpretation eines literarischen Werkes unverzichtbar sind;

- Kenntnis und Anwendung der interkulturellen Kompetenz (Fremdheitskompetenz), d. h. des eigenen und fremden kulturellen Codes;

- Fähigkeit zu einem bikulturellen Gespräch und transkulturellen Vergleichen, die eine Voraussetzung für das fremdkulturelle Verstehen schaffen.

Das letzte Ziel, das fremdkulturelle Verstehen, scheint allerdings besonders schwer erreichbar zu sein, weil es von den Lernern nicht nur eine kognitive, sondern auch eine affektive Bereitschaft erfordert, das Fremde zu verstehen zu versuchen. Das Verstehen fremdkultureller Erscheinungen, darunter auch literarischer Texte, wird nicht dadurch erreicht, dass man die jeweilige fremde Identität annimmt. Es geschieht eben nur durch die Fähigkeit, den interkulturellen Dialog aufnehmen zu können (Karolak, 1999, S. 39). Daher ist es ein hoch komplizierter und mehrgliedriger Prozess, der aus mindestens folgenden vier Phasen besteht:

(1) Reflexion über die fremde Zielkultur (auf die man sich hinbewegt) als auch der eigenen Kultur, weil sie aus dieser Perspektive der Ausgangskultur kennen gelernt wird. Die Wahrnehmung der fremden Zielkultur ist dabei oft von Stereotypen und stereotypenähnlichen Vorstellungen sowie Vorurteilen geprägt, wodurch das interkulturelle Lernen im Ausgangspunkt bedroht ist;

(2) Begegnung mit der jeweiligen fremden Kultur und ihren Komponenten. Im ersten Moment bewirkt sie negative Emotionen und Einstellungen, wie etwa Beunruhigung, Entfremdungsgefühl bis hin zur Gefährdung, weil das Fremde/das Unbekannte das bisherige Weltbild sehr oft in Frage stellt und deshalb starke Abwehrmechanismen auslöst. Gleichzeitig beginnt jedoch die affektive Kompensation, weil das Neue und das Unbekannte dank ihrer Anziehungskraft unser Interesse wecken und uns verführen;

(3) Auseinandersetzung und Zusammenspiel der beiden Kulturen: der eigenen und der fremden. Auf dieser Etappe agieren überwiegend kognitive Faktoren und lassen uns unsere Erfahrungen im eigenen Kulturkreis mit neuen Informationen und Erfahrungen vergleichen, gegenseitig aufeinander beziehen und uns mit ihnen auseinandersetzen;

(4) Neuformulierung und Neupositionierung beider Kulturen. In dieser Phase werden beide Bilder, das der neuen und auch der eigenen Kultur, umstrukturiert und neu geprägt. Scheitert das fremdkulturelle Verstehen (je nach den Umständen, unter welchen der Prozess verläuft), so wird das stereotype Bild 
der fremden Kultur bestätigt oder sogar verstärkt. Ein erfolgreiches fremdkulturelles Verstehen bewirkt dagegen, dass das Bild der fremden Kultur relativiert und objektiviert wird. Damit jedoch eine neue Größe, neue Erfahrungsbildung wirksam sein könnte, muss auch die eigene Kultur neu formuliert und neu positioniert werden. Dieses bedeutet keinesfalls ihre Verarmung, sondern eine wesentliche Bereicherung.

Das fremdkulturelle Verstehen kann nur dann gelingen, wenn fremdsprachige Leser auf den interkulturellen Dialog entsprechend vorbereitet werden, indem sie alle oben genannten Komplexziele realisieren. Die Öffnung der Perspektive der eigenen Kultur ist allerdings eine äußerst schwere Aufgabe, weil sie zugleich eine kognitive und affektive Leistung des Lesers erzwingt. Wird ein fremdsprachiger Leser diese Bemühung aufwenden, so gewinnt er auch die Chance, dass der fremde kulturelle Code nicht nur rezeptiv wahrgenommen wird, sondern auch dafür, dass dieser in sein kognitives Verarbeitungspotenzial eingebaut und mit ihm integriert wird. Auf diese Art und Weise erweitert er auch die Grenzen seiner Kognition in beiden Sprachen: in der Mutter- und der Fremdsprache. Ein fremdsprachiger Leser kann demnach den interkulturellen Dialog auch für eine Bereicherung der eigenen Kultur verwerten.

Der kulturelle Code ist leider in virtuellen (didaktischen) Bedingungen nur bedingt und begrenzt erlernbar, wodurch auch das fremdkulturelle Verstehen nicht immer vollkommen gelingen wird. Die Aufgabe der Literaturdidaktik in fremdkultureller Perspektive besteht gerade darin, solche Bedingungen im Literaturunterricht zu schaffen, damit die Auseinandersetzung mit dem Text zu einer möglichst authentischen Erfahrung der Fremde werden kann. Nur dann wird fremdsprachige Lektüre zu dem Erlebnis, das Gadamer folgendermaßen schildert:

Wo Verständigung ist, da wird nicht übersetzt, sondern gesprochen. Eine fremde Sprache verstehen bedeutet ja, sie nicht in die eigene Sprache übersetzen müssen. Wo einer eine Sprache wirklich beherrscht, bedarf es keiner Übersetzung mehr, ja erscheint jede Übersetzung unmöglich. Eine Sprache verstehen ist selbst noch gar kein wirkliches Verstehen und schließt keinen Interpretationsvorgang ein, sondern ist ein Lebensvollzug (1975, S. 362).

Eine interkulturelle Texthermeneutik, deren einige Komponenten in diesem Beitrag skizziert wurden, mag diesem Anspruch von Gadamer gerecht werden.

\section{Bibliographie}

Barkowski, H. \& Krumm, H.-J. (2010). Fachlexikon Deutsch als Fremd-und Zweitsprache. Tübingen: Francke.

Ehlers, S. (1998). Lesetheorie und fremdsprachliche Lesepraxis aus der Perspektive des Deutschen als Fremdsprache. Tübingen: Gunter Narr. 
Gadamer, H. G. (1960). Wahrheit und Methode. Grundzüge einer philosophischen Hermeneutik. Tübingen: Mohr.

Gadamer, H. G. (2003). Język i rozumienie. Warszawa: Fundacja Aletheia.

Habermas, J. (1986). Uniwersalistyczne roszczenie hermeneutyki. In H. Orłowski (Hrsg.), Współczesna myśl literaturoznawcza w Republice Federalnej Niemiec. Antologia (S. 36-60).Warszawa: Czytelnik.

Iser, W. (1976). Der Akt des Lesens. Theorie ästhetischer Wirkung. München: Fink.

Karolak, Cz. (1996). Literaturverstehen und Textdidaktik in fremdsprachenspezifischer Perspektive. Convivium. Germanistisches Jahrbuch Polen, 345-358.

Karolak, Cz. (1999). Dydaktyka literatury wobec potrzeb nauki języka w warunkach obcokulturowych. Poznań: Wydawnictwo Naukowe UAM.

Karolak, Cz. (2004). Zur integrierenden Funktion von Literatur im Verstehensprozess fremder Kultur. In C. Badstübner, R. Rozalowska-Żądło \& A. Uniszewska (Hrsg.), Sprachen lehren. Sprachen lernen (S. 231-239). Gdańsk: Wydawnictwo Uniwersytetu Gdańskiego.

Krusche, D. (1981). Lese-Unterschiede. Zum interkulturellen Lesergespräch. Jahrbuch Deutsch als Fremdsprache, 7, 1-17.

Krusche, D. (1995). Leseerfahrung und Lesegespräch. München: Iudicium.

Steinmetz, H. (1996). Aneignung. Eine brauchbare Kategorie für den Umgang mit literarischer Fremdheit. In A. Wierlacher \& G. Stötzel (Hrsg.), Blickwinkel. Kulturelle Optik und interkulturelle Gegenstandskonstitutionen. Akten des III. Internationalen Kongresses der Gesellschaft für Interkulturelle Germanistik Düsseldorf 1994 (S. 443-451). München: Iudicium.

Szeluga, A. (2002). Zur Konvergenz der Lehrziele der Sprach- und der Literaturdidaktik auf der Ebene des Textverstehens. Studia Germanica Posnaniensia, XXVIII, 195-207.

Szeluga, A. (2003a). Pragmalinguistische Komponenten der kommunikativen Kompetenz in fremdkultureller Perspektive. In L. W. Zybatow (Hrsg.), Linguistik International. Europa der der Sprachen: Sprachkompetenz-Mehrsprachigkeit-Translation. Teil I: Sprache und Gesellschaft. Akten des 35. Linguistischen Kolloquiums in Innsbruck 2000 (S. 411- 417). Frankfurt a. M.: Peter Lang.

Szeluga, A. (2003b). Eigene und fremde kulturelle Metaphern. Ausgewählte Aspekte fremdsprachlicher Textrezeption aus kognitiver Sicht. Studia Niemcoznawcze, XXVI, 839-847.

Szeluga, A. (2004a). Brauchen wir eine Didaktik der Fremdheitskompetenz? Multikulturelle Faktoren im modernen Fremdsprachenunterricht. Studia Niemcoznawcze, XXVIII, 945-952.

Szeluga, A. (2004b). Sprachliche vs. linguistische Kommunikation im Text und seinen Strukturen. Ein Beitrag zur Erforschung der Intertextualität. Studia Niemcoznawcze, XXVII, 653-664.

Szeluga, A. (2007). Interkulturelle Texthermeneutik als Subdisziplin der modernen Literaturdidaktik. In E. Białek \& Cz. Karolak, ,Schuhnummer oder Leben : Beiträge zur Literaturdidaktik und zum kinder- und jugendliterarischen Schrifttum (S. 42-50). Wrocław: Atut.

Volkmann. L. (2002). Aspekte und Dimensionen interkultureller Kompetenz. In L. Volkmann, K. Stierstoffer, W. Gehring (Hrsg.), Interkulturelle Kompetenz. Konzepte und Praxis des Unterrichts (S. 11-47). Tübingen: Gunter Narr. 